\title{
La liaison variable dans un corpus du français méridional : L'importance relative de la fonction grammaticale
}

\author{
Diana Ranson \\ Université de Géorgie \\ dranson@uga.edu
}

\section{La liaison variable et le problème de la diversité}

La liaison, définie comme la réalisation d'une consonne latente devant un mot commençant par une voyelle, comme la prononciation [iletale] pour il est allé, varie selon des facteurs de toutes sortes: linguistiques, diastratiques, diaphasiques, diatopiques, et diachroniques. Cette diversité de facteurs, aussi intéressante soit-elle, a engendré à son tour une multiplicité de résultats et d'interprétations de la part des linguistes (Green et Hintze 1990 :71). Cet article poursuit donc l'objectif de déterminer l'importance relative de deux facteurs linguistiques et deux facteurs diastratiques sur la liaison variable dans un corpus du français méridional et poursuit ainsi la suggestion de Booij et De Jong (1987:1015) que les recherches futures déterminent le degré d'influence des facteurs sur la liaison variable. Comme point de départ nous proposons que ce soit la fonction grammaticale (autrement dit le lien syntaxique) des mots en liaison qui a le plus grand effet sur sa réalisation. En outre nous proposons qu'il soit plus révélateur de regrouper les liaisons variables selon la fonction grammaticale dans les analyses des autres facteurs, comme par exemple les facteurs diastratiques $\mathrm{du}$ sexe et de l'âge. Le fait de ne pas distinguer entre fonctions grammaticales peut être une cause des résultats divers dans les études antérieures diverses sur ce phénomène linguistique.

Un premier point de diversité dans les études antérieures est bien le choix des liaisons à analyser. Certaines études ont tenu compte des liaisons invariables aussi bien que les variables (Malécot 1975, Green et Hintze 1990, Pagliano et Laks 2005, Durand et Lyche 2008) tandis que d'autres n’ont considéré que les liaisons variables (Ågren 1973; Ashby 1981; Encrevé 1988; De Jong 1991 ; Armstrong 1993 ; Smith 1996, 1998). Selon l'approche variationniste c'est cette dernière posture qu'on devrait adopter puisque l'inclusion des contextes catégoriques dans les analyses de la variation risque de fausser les résultats (Tagliamonte $2006: 13$ ). Mais les définitions des liaisons invariables et des liaisons variables varient à leur tour dans les études antérieures et dans les manuels du français. Citons à titre d'exemple la classification de la liaison après une préposition comme dans ou chez ou après un adverbe monosyllabique comme très ou bien comme invariable par Delattre 1947 (cité par Encrevé 1988 :48) et par Valdman (1993) et comme variable par Encrevé (1988:47) et par Lodge et al. (1997 :99). La solution adoptée dans cette étude face à la diversité de classifications, et aussi celle adoptée par Pagliano et Laks (2005), est de classer les liaisons selon leur variabilité dans un corpus spécifique du français parlé.

Un deuxième point de diversité s'ensuit du premier. Les différences entre les liaisons analysées mèneront à des résultats différents. Prenons l'exemple du taux de liaison réalisée pour les différentes consonnes de liaison, c'est-à-dire les consonnes latentes qui sont prononcées lors de la réalisation d'une liaison. On ne s'attend pas à ce que les consonnes de liaison se placent dans le même ordre selon le taux de liaison réalisée dans une analyse des liaisons invariables et variables que dans une analyse des liaisons variables seulement. Or, on constate que Pagliano et Laks (2005 :5), qui ont analysé toutes les sortes de liaison, ont trouvé l'ordre [z], [n], [t] pour le taux de liaison selon la consonne latente tandis qu'Ashby (1981:50), qui n'a analysé en principe que les liaisons variables, a trouvé l'ordre [n], [t], [z]. Durand et Lyche (2008:58-59), qui comme Pagliano et Laks ont analysé les liaisons invariables aussi bien que les variables, ont trouvé le même ordre pour le nombre de liaisons réalisées pour chaque consonne de liaison, c'est-à-dire [z] (4544), [n] 
(3689), et [t] (1665), sans toutefois calculer le taux de réalisation. Une explication possible pour ces ordres différents est que le taux de liaison du [z], la plus grande différence entre les résultats des études, est augmenté dans les études de Pagliano et Laks (2005) et de Durand et Lyche (2008) par son apparence fréquente dans des liaisons invariables, comme les amis, mes amis, nous aimons, et ils aiment. Si on pouvait le confirmer, on s'attendrait à constater que le taux de liaison pour la consonne latente dans ces études dépendait directement de la fonction grammaticale des mots en liaison. Ce qui est moins évident pourtant est que les différentes proportions des mots en liaison dans un corpus dues au hasard pourraient provoquer des résultats différents aussi. Comment expliquer alors pourquoi une autre étude (Malécot 1975 :164), qui a analysé toutes les sortes de liaison tout comme celles de Pagliano et Laks (2005) et Durand et Lyche (2008), a trouvé l'ordre $[\mathrm{n}],[\mathrm{z}],[\mathrm{t}]$ au lieu de $[\mathrm{z}],[\mathrm{n}],[\mathrm{t}]$ ? Une possibilité est que le renversement du $[\mathrm{z}]$ et du $[\mathrm{n}]$ dans ces deux études soit le résultat de différentes proportions des liaisons invariables en [n], comme un ami, mon ami, on aime, $j$ 'en achète, et en [z] dans les deux corpus. Si c'était le cas, ce serait encore une raison d'insister sur l'importance de la fonction grammaticale comme facteur pour la liaison variable. La même situation pourrait se présenter aussi dans les études qui ne tiennent compte que des liaisons variables. Supposons que par hasard le [z] apparaît plus souvent dans un corpus dans des expressions variables où la liaison est souvent faite, comme très important, que dans des expressions où elle n'est pas souvent faite, comme pas encore, mais que le contraire arrive au [t] qui apparaît plus souvent dans une expression comme il fait un appel que dans une expression comme $i l$ est allé. Alors, on risquerait de tirer la conclusion qu'une certaine consonne favorise la liaison, mais en réalité c'est la fonction grammaticale qui est déterminante. Bien que nous n'ayons pas pu refaire les calculs des études antérieures, nous mettrons cette idée à l'épreuve par la confection d'un tableau croisé qui montre le taux de liaison pour chaque consonne latente selon la fonction grammaticale. En plus nous soumettrons les données à une analyse multivariable à l'aide du logiciel Goldvarb X (Sankoff, Tagliamonte, et Smith 2005) pour déterminer le poids relatif et la signifiance statistique de chacun des facteurs analysés - la classe grammaticale, la consonne latente, le sexe et l'âge des locuteurs - et l'interaction entre eux. ' (Tagliamonte 2006 :217-34 décrit cette méthode.)

Ce problème de proportion inégale de liaison de fonctions grammaticales différentes peut affecter aussi la corrélation entre les caractéristiques sociales des locuteurs et la réalisation de la liaison. Malécot (1975 : 169), par exemple, a trouvé un plus haut taux de liaison invariable et variable réalisée par les femmes que par les hommes (67\% contre 62\%) tout comme De Jong et al. (1981, cité par Booij et De Jong 1987 :1017) pour quelques liaisons variables, tandis qu'Ashby (1981: 54) a trouvé un taux de liaison variable plus élevé chez les hommes (36\% contre $32 \%)$. En ce qui concerne l'âge des locuteurs, Ashby (1981:54) a trouvé que le taux de liaison réalisée montait avec l'âge, une idée soutenue aussi par De Jong et al. (1981, cité par Booij et De Jong $1987: 1017$ ). Spécifiquement, dans l'étude d'Ashby, les jeunes de 14 à 21 ans ont fait moins de liaisons que les personnes de 51 à 64 ans $(30 \%$ contre 37\%), mais Malécot $(1975: 169)$ n'a pas trouvé de patron net selon l'âge. Le taux de liaisons faites par les plus jeunes de 20 à 29 ans $(63 \%)$ était supérieur à celui des locuteurs de 30 à 39 ans $(59 \%)$ et le taux pour les gens de 50 à 59 ans (69\%) était légèrement plus élevé que celui pour les locuteurs encore plus âgés, de 60 à 69 ans (68\%). Bien que la possibilité d'une différence due à la fonction grammaticale des mots en liaison ne soit pas aussi évidente pour les facteurs diastratiques, comme le sexe et l'âge, que pour un facteur linguistique, comme la consonne de liaison, la fonction grammaticale pourrait toujours jouer un rôle. Si par hasard les hommes ou les vieux produisaient un plus grand nombre d'une certaine fonction grammaticale de liaison que les femmes ou les jeunes, les résultats feraient penser que cette différence est due au sexe ou à l'âge des locuteurs sans que ce soit vraiment le cas. Notre solution à ce problème sera la même que celle proposée pour la consonne de liaison. Nous ferons un tableau croisé qui montre le taux de liaison pour chaque sexe et âge selon la fonction grammaticale des mots en liaison et nous soumettrons ces facteurs à une analyse multivariable à l'aide de Goldvarb X. Avant de présenter les résultats dans des tableaux croisés, nous décrirons nos méthodes et nous présenterons le taux de liaison pour chaque fonction grammaticale séparément avant de le combiner avec d'autres facteurs. 


\section{Méthodes}

\subsection{Le corpus}

Une étude sur un phénomène de la parole comme la liaison variable doit se baser sur un corpus du français parlé. La présente étude analyse donc la parole de 16 locuteurs natifs du français du sud de la France, dont le Tableau 1 présente les caractéristiques sociales. ${ }^{2}$

Tableau 1 : Caractéristiques sociales des sujets parlants et nombre de sites de liaison

\begin{tabular}{|c|c|c|c|c|c|}
\hline & $\begin{array}{l}\text { locuteur } \\
\text { (sites de } \\
\text { liaison) }\end{array}$ & âge & études & profession & $\begin{array}{l}\text { lieu } \\
\text { d'origine }\end{array}$ \\
\hline \multirow{4}{*}{$\begin{array}{l}\text { jeunes } \\
\text { femmes }\end{array}$} & A (89) & 25 & $\mathrm{bac}+4$ & enseignante du FLE & la Lozère \\
\hline & $\mathrm{B}(57)$ & 25 & $\mathrm{bac}+4$ & étudiante en FLE & Nîmes \\
\hline & $\mathrm{C}(68)$ & 32 & $\mathrm{bac}+4$ & chargée de cours du FLE & Béziers \\
\hline & $\mathrm{D}(60)$ & 22 & $\mathrm{bac}+2$ & spécialiste de l'événementiel & Rognes \\
\hline \multirow{4}{*}{$\begin{array}{l}\text { jeunes } \\
\text { hommes }\end{array}$} & E (80) & 25 & $\mathrm{bac}+3$ & étudiant en chimie & Montpellier \\
\hline & $\mathrm{F}(89)$ & 28 & $\mathrm{bac}+8$ & étudiant en électronique & Narbonne \\
\hline & G (107) & 24 & $\mathrm{bac}+2$ & étudiant en horticulture & Martigues \\
\hline & $\mathrm{H}(84)$ & 26 & $\mathrm{bac}+2$ & inspecteur de raffineries de pétrole & Rognes \\
\hline \multirow{4}{*}{$\begin{array}{l}\text { femmes } \\
\text { âgées }\end{array}$} & S (89) & 55 & $\mathrm{bac}+5$ & enseignante du FLE & Narbonne \\
\hline & $\mathrm{T}(116)$ & 56 & sans bac & retraitée de la SNCF & Montpellier \\
\hline & $\mathrm{U}(62)$ & 53 & sans bac & nourrice & Rognes \\
\hline & $\mathrm{V}(111)$ & 40 & $\mathrm{bac}+3$ & bibliothécaire & Avignon \\
\hline \multirow{4}{*}{$\begin{array}{l}\text { hommes } \\
\text { âgés }\end{array}$} & W (104) & 56 & $\mathrm{bac}+5$ & fonctionnaire à la DCCRF & Béziers \\
\hline & $\mathrm{X}(122)$ & 65 & $\mathrm{bac}+5$ & enseignant du FLE & Conques \\
\hline & $\mathrm{Y}(95)$ & 55 & sans bac & fermier & Rognes \\
\hline & Z (85) & 55 & $\mathrm{bac}+2$ & boucher & Marseille \\
\hline
\end{tabular}

Les locuteurs sont répartis en quatre groupes selon le sexe et l'âge, avec un âge moyen de 26 ans pour les jeunes et de 54 ans pour les vieux. Le niveau d'études ainsi que la profession de chaque locuteur sont indiqués dans le souci de fournir des renseignements complets mais il n'est pas possible de tester le niveau d'études comme facteur à part. La plupart des locuteurs ont un niveau d'études similaire alors il est impossible de former deux groupes de locuteurs qui sont similaires en sexe et âge mais différents en niveau d'études. Il est intéressant de noter tout de même que les jeunes, avec une moyenne de bac $+3,6$ ont un niveau d'études supérieur à celui des vieux, avec une moyenne de bac $+2,5$. En tout douze conversations ont été analysées. Quatre ont eu lieu entre l'auteure et un couple (locuteurs B et F, D et H, S et W, et U et Y) et huit entre l'auteure et un seul locuteur. Sept de ces conversations ont été enregistrées en juillet 2005 à Montpellier, celles avec les locuteurs A, B, C, E, F, S, T, W, et X, et cinq ont eu lieu à Rognes en mai 2006, avec les locuteurs $\mathrm{D}, \mathrm{G}, \mathrm{H}, \mathrm{U}, \mathrm{V}, \mathrm{Y}$ et $\mathrm{Z}$. Pour la présente étude 15 à 40 minutes de chaque conversation ont été transcrites et analysées dans le but d'analyser un minimum de 60 sites de liaison par locuteur, dont le nombre est indiqué pour chaque locuteur après son identifiant.

\subsection{Analyse}

Dans un premier temps, nous avons noté tous les sites possibles de liaison variable sur la transcription écrite des conversations selon les fonctions grammaticales mentionnées dans la littérature. Ces liaisons, proposées par Delattre $(1966: 43)$ et ensuite reproduites parfois avec des modifications par Encrevé (1993: 47), Valdman (1993: 150-76), Lodge et al. (1997: 99), et Armstrong (2001 : 182), comprennent un nom pluriel suivi d'un adjectif (fraudes économiques) ou d'un verbe (les ânes étaient passés), un verbe suivi d'une autre forme verbale (on est arrivé, ils vont 
insister) ou d'un complément (ils arrivaient à la maison), et un mot invariable monosyllabique (très important, quand il y a du blé) ou polysyllabique (assez importantes, j'ai toujours eu) suivi par n'importe quel mot commençant par une voyelle. Dans un deuxième temps nous avons écouté les enregistrements des conversations pour déterminer si les locuteurs avaient fait chaque liaison ou non, mais nous n'avons pas compté les sites possibles interrompus par une pause ou la voyelle d'hésitation $e u h .^{3}$ Nous avons remarqué pendant l'écoute qu'une liaison considérée typiquement comme invariable était en réalité variable et donc nous avons décidé de l'inclure dans l'analyse des liaisons variables. L'expression figée c'est-à-dire est prononcée tantôt comme [setadiR], tantôt comme [seadiR] ou même [sadiR], comme Green et Hintze (1990 :73) ont remarqué également. On a constaté dans notre corpus que les locuteurs n'ont jamais réalisé quelques liaisons considérées typiquement comme variables : la liaison après les conjonctions alors, après, mais, et puis, après les adverbes polysyllabiques assez, beaucoup, certainement, également, éventuellement, finalement, justement, longtemps, maintenant, quasiment, souvent, surtout, tellement, totalement, et toujours, et, à notre plus grande surprise, après les adverbes négatifs pas et jamais. Nous avons choisi de ne pas exclure ces classes grammaticales de la comparaison de nos résultats avec ceux des études antérieures puisque ces autres études les avaient considérées. Pourtant dans l'analyse variationniste de nos données par Goldvarb X, nous mettrons de côté toutes les liaisons qui sont catégoriques dans notre corpus. ${ }^{4}$

\section{Résultats et discussion}

Par la présentation des résultats de la réalisation de la liaison variable dans notre corpus nous testerons l'hypothèse que la fonction grammaticale des mots en liaison est le facteur principal dans sa réalisation. D'abord nous verrons le taux de réalisation pour chaque fonction grammaticale et ensuite nous verrons l'interaction de ce facteur avec le facteur linguistique de la consonne de liaison et finalement nous verrons son interaction avec les facteurs diastratiques du sexe et de l'âge.

\subsection{La liaison variable et la fonction grammaticale}

Le Tableau 2 ci-dessous présente le taux de réalisation de la liaison variable en ordre décroissant de sa réalisation dans notre corpus en comparaison avec les résultats des autres études. Le chiffre qui apparaît entre parenthèses après le nom de la fonction grammaticale est le poids de chaque facteur calculé par Goldvarb X. Un chiffre supérieur à 0,500 indique que cette classe grammaticale favorise la réalisation de la liaison tandis qu'un chiffre plus bas indique que la liaison est défavorisée par cette construction. Ce chiffre est absent pour les deux dernières catégories du tableau, l'adverbe négatif et les conjonctions alors, après, mais et puis, parce qu'elles ont été exclues de l'analyse en raison de la non réalisation catégorique de la liaison. ${ }^{5}$ La classe grammaticale s'est avérée très significative statistiquement au niveau de moins de 0,0001, un premier indice de son importance.

Bien qu'il y ait des différences notables entre les taux de liaison réalisée entre les différentes études, si on lit dans chaque colonne de haut en bas, on trouve que certaines liaisons sont très souvent faites tandis que d'autres ne sont presque jamais faites. En plus on constate pour chaque étude de très grandes différences entre le taux de liaison pour la fonction grammaticale qui favorise la liaison le plus et celle qui la favorise le moins. Pour la présente étude on trouve un écart de 96; on trouve 87 et 81 respectivement pour Malécot (1975) et Ashby (1981), et seulement 65 et 66 respectivement pour Ågren (1973) et Smith (1996). Il y a un écart moins grand dans ces deux dernières études peutêtre parce que leurs auteurs ont présenté des chiffres pour moins de fonctions grammaticales et parce que leur corpus consistait des enregistrements des émissions de radio dans lesquelles on trouverait un plus haut taux de liaison que dans les conversations familières. On constate aussi une grande différence entre le poids de chaque facteur qui descend de 0,986 jusqu'à 0,075 . Ces chiffres montrent bien l'importance indéniable de la fonction grammaticale pour la réalisation de la liaison. 
Tableau $2:$ Le taux de liaison réalisée selon la fonction grammaticale du mot liaisonnant ${ }^{6}$

\begin{tabular}{|c|c|c|c|c|c|}
\hline & $\begin{array}{l}\text { présente } \\
\text { étude }\end{array}$ & $\begin{array}{l}\text { Ågren } \\
1973^{\mathrm{a}} \\
\end{array}$ & $\begin{array}{l}\text { Malécot } \\
1975: 164\end{array}$ & $\begin{array}{l}\text { Ashby } \\
1981: 50\end{array}$ & $\begin{array}{l}\text { Smith } \\
1996^{\mathrm{b}}\end{array}$ \\
\hline adv. très, plus $(0,986)$ & $49 / 51$ & & $111 / 176$ & & \\
\hline très important & $96 \%$ & & $63 \%^{\mathrm{c}}$ & & \\
\hline préposition $(0,981)$ & $88 / 93$ & & $168 / 182$ & $25 / 51$ & \\
\hline dans une famille & $95 \%$ & & $92 \%$ & $49 \%$ & \\
\hline être (aux.) $(0,890)$ & $33 / 45$ & & $30 / 43$ & & \\
\hline il est arrivé & $73 \%$ & & $70 \%$ & & \\
\hline quand (conj.) $(0,869)$ & $36 / 52$ & & & $119 / 142$ & \\
\hline quand elle téléphone & $69 \%$ & & $100 \%{ }^{\mathrm{c}}$ & $83 \%$ & \\
\hline c'est-à-dire $(0,673)$ & $7 / 17$ & & & & \\
\hline & $41 \%$ & & & & \\
\hline être (copule) $(0,508)$ & $98 / 377$ & & $366 / 509$ & $331 / 681$ & \\
\hline c'est une langue & $26 \%$ & & $72 \%$ & $48 \%$ & \\
\hline nom pl. + adj./verb $(0,309)$ & $7 / 53$ & $170 / 639$ & $9 / 50$ & $11 / 102$ & $68 / 309$ \\
\hline étudiants étrangers & $13 \%$ & $27 \%$ & $18 \%$ & $11 \%$ & $22 \%$ \\
\hline avoir (aux.) $(0,286)$ & $3 / 25$ & $586 / 1140$ & $8 / 49$ & & $115 / 411$ \\
\hline ils ont été & $12 \%$ & $51 \%{ }^{\mathrm{d}}$ & $16 \%$ & & $28 \% \%^{\mathrm{d}}$ \\
\hline adv. polysyllabique ${ }^{\mathrm{e}}$ & $7 / 95$ & $395 / 988$ & $5 / 98$ & & $30 / 234$ \\
\hline$(0,580)$ ça a toujours été & $7 \%$ & $40 \%$ & $5 \%$ & & $13 \%$ \\
\hline verbe $(0,075)$ & $8 / 372$ & & $11 / 210$ & $39 / 515$ & \\
\hline il y avait un animal & $2 \%$ & & $5 \%{ }^{\mathrm{f}}$ & $7 \%$ & \\
\hline adv. négatif & $0 / 76$ & $222 / 965$ & $16 / 43$ & $24 / 142$ & $81 / 379$ \\
\hline pas informatisé & $0 \%$ & $23 \% \%^{\mathrm{g}}$ & $37 \%{ }^{\mathrm{g}}$ & $16 \%$ & $21 \%^{\mathrm{g}}$ \\
\hline alors, après, mais, puis & $0 / 152$ & & & $16 / 726$ & \\
\hline mais après, puis ensuite & $0 \%$ & & & $2 \%$ & \\
\hline
\end{tabular}

${ }^{a}$ cité par Booij et De Jong $1987: 1012$ et Armstrong $2001: 197$

bité par Armstrong $2001: 197$

${ }^{\mathrm{c}}$ Malécot (1975 : 166) calcule le taux de liaison pour tous les adverbes monosyllabiques. Il précise que la liaison est obligatoire après quand sans donner le nombre d'occurrences.

davoir auxiliaire et lexical

epour le présent corpus cette catégorie comprend aussi les adverbes bien, mieux et moins. Le taux de liaison pour les adverbes polysyllabiques tout seuls est $0 \%(0 / 79)$.

f verbes principaux exceptés être et avoir

${ }^{\mathrm{g}}$ seulement pas

Les liaisons variables qui sont presque toujours faites dans la présente étude ont lieu après l'adverbe très ou plus ou après une préposition. ${ }^{7} \mathrm{Il}$ est intéressant que la liaison se fasse encore plus après une 
préposition dans ce corpus que ce n'était le cas il y a environ 30 ans dans le corpus de Malécot et celui d'Ashby. Elle se fait plus aussi après un adverbe monosyllabique dans notre corpus que dans le corpus de Malécot. Même si on considère les adverbes monosyllabiques bien, mieux et moins en plus de très et plus, le taux de liaison de 75\% (57/76) est supérieur au taux de 63\% dans le corpus de Malécot. On voit que le taux de liaison après être comme auxiliaire a légèrement augmenté aussi ce qui nous mène à l'observation que le taux de réalisation des liaisons les plus souvent faites est plus élevé dans notre étude que dans les études antérieures. Il faut tout de même tenir en compte l'origine méridionale de nos locuteurs. Durand et Lyche (2008:47-48) ont trouvé un plus haut taux de liaison chez les locuteurs du sud que chez ceux du nord. Spécifiquement le taux de liaison chez les locuteurs du sud était de $43 \%$ (146/342) après c'est contre 34\% (155/457) chez les locuteurs du nord. Les chiffres pour la liaison après c'était $(16 \%$ contre $5 \%)$ et avait $(11 \%$ contre $0 \%)$ ont suivi le même patron. Alors la différence entre nos résultats et ceux des études antérieures pourrait être aussi bien géographique que chronologique.

Pourtant on constate une baisse considérable du taux de liaison dans d'autres catégories grammaticales, notamment après être comme copule qui était de $72 \%$ dans l'étude de Malécot, $48 \%$ dans l'étude d'Ashby et qui n'est que $26 \%$ dans la présente étude. De la même façon la conjonction quand est passée d'un statut invariable dans le corpus de Malécot à un taux de $87 \%$ chez Ashby jusqu'à son taux actuel de 69\%. La liaison dans l'expression c'est-à-dire qui, on suppose, était considérée comme invariable dans le passé, n'est réalisée que dans $41 \%$ de ces occurrences. La baisse du taux de liaison est aussi notable après pas qui descend de $16 \%$ chez Ashby jusqu'à $0 \%$ chez nous. Les autres catégories variables de liaison avaient dans le passé et ont toujours un faible taux de liaison. La liaison est réalisée après 7 noms seulement sur 53 dans le présent corpus mais tous sont dans des expressions qu'on pourrait qualifier de figées. L'expression personnes âgées est prononcée avec liaison une fois par le locuteur $\mathrm{H}$ et deux fois par $\mathrm{T}$. Le locuteur $\mathrm{X}$ dit une fois affaires étrangères et trois fois étudiants étrangers en prononçant la consonne latente. Autrement la liaison ne se fait pas après un nom suivi d'un adjectif et elle ne se fait jamais entre nom et verbe. La liaison après l'auxiliaire avoir se fait seulement trois fois : une fois par le locuteur X dans ils ont été et deux fois par la locutrice $\mathrm{S}$ dans ils avaient été. Des 8 liaisons réalisées après un verbe, une a été faite par le locuteur X, une autre par W et les autres 6 par la locutrice S. Et finalement la liaison ne se fait plus du tout après un adverbe polysyllabique ni après une conjonction comme après, avant, mais, ou puis. En fin de compte il reste seulement quelques catégories selon la fonction grammaticale où la liaison continue à être variable en termes pratiques: après la conjonction quand et après être qu'il soit employé comme auxiliaire, copule ou dans l'expression c'est-à-dire.

Pour résumer on peut dire que la liaison variable selon la classe grammaticale devient moins variable. Elle se fait encore plus dans les trois catégories où elle était déjà fréquente et elle se fait encore moins dans les catégories où elle ne se faisait que rarement. Même pour les catégories qui sont restées variables on constate un taux de liaison plus bas que dans le passé.

\subsection{La liaison variable et la consonne de liaison}

Des études antérieures ont noté la corrélation entre un facteur linguistique, la consonne de liaison, et la réalisation de la liaison variable (Malécot 1975 :168, Ashby 1981 :50, Encrevé 1988 :68, Booij et De Jong 1987 :1013, Durant et Lyche 2008 :58-59). Nous cherchons ici à déterminer si la consonne de liaison est un facteur important à elle seule où si elle dépend plutôt de la fonction grammaticale des mots en liaison. On constate d'abord que la consonne latente est statistiquement significative à un niveau de moins de 0,0001 tout comme la classe grammaticale. Le Tableau 3 présente les trois consonnes latentes suivies du poids et du taux de liaison pour chaque consonne dans la présente étude et trois études antérieures. Toutes les études, à l'exception d'Encrevé (1988:77-78, note 11), trouvent le plus haut taux de liaison pour [n], bien que le nombre d'occurrences du [n] soit plus petit que celui des consonnes [z] et [t]. Dans l'étude de Malécot la consonne [z] favorise la liaison plus que le [t]. Cela est vrai aussi dans notre corpus. Quand on exclut les liaisons catégoriques, le taux de 
liaison pour le [z] est de $43 \%$ contre $24 \%$ pour le [t] et le poids indique que le [z] favorise la liaison et que le [t] la défavorise. Dans les études d'Ashby et Encrevé l'ordre est renversé ; le [t] favorise la liaison plus que le [z]. Pour expliquer cette différence, il faut se rappeler que Malécot a analysé les liaisons invariables en plus des variables, alors le taux plus élevé de [z] pourrait être dû à son emploi dans des liaisons telles que les amis, je les aime, et ils aiment. Dans notre corpus le fait d'écarter les liaisons jamais réalisées a eu le même effet, alors il y avait sans doute un plus grand nombre d'exclusions avec la consonne [z] qu'avec le [t]. Une autre possibilité serait simplement que les locuteurs des différents corpus ont choisi de faire différentes liaisons, mais il reste toujours à savoir si cela est dû à la consonne de liaison elle-même où plutôt à la classe grammaticale du mot liaisonnant.

Tableau 3 : Le taux de liaison réalisée selon la consonne latente

\begin{tabular}{|l|l|l|l|l|l|l|}
\hline $\begin{array}{l}\text { consonne } \\
\text { latente }\end{array}$ & $\begin{array}{l}\text { présente } \\
\text { étude } A^{\mathrm{a}}\end{array}$ & $\begin{array}{l}\text { présente } \\
\text { étude B }\end{array}$ & $\begin{array}{l}\text { Malécot } \\
1975: 168\end{array}$ & $\begin{array}{l}\text { Ashby } \\
1981: \\
50 \mathrm{~A}^{\mathrm{c}}\end{array}$ & $\begin{array}{l}\text { Ashby } \\
1981: \\
50 \mathrm{~B}^{\mathrm{c}}\end{array}$ & $\begin{array}{l}\text { Encrevé } \\
1988: 68^{\mathrm{a}}\end{array}$ \\
\hline$[\mathrm{n}](0,916)$ & $46 / 55$ & $46 / 55$ & $726 / 769$ & $127 / 205$ & $10 / 24$ & $\mathrm{c}$ \\
& $84 \%$ & $84 \%$ & $94 \%$ & $61 \%$ & $42 \%$ & \\
\hline$[\mathrm{z}](0,617)$ & $122 / 531$ & $122 / 282$ & $148 / 2447$ & $389 / 1363$ & $263 / 1205$ & \\
& $23 \%$ & $43 \%$ & $61 \%$ & $28 \%$ & $22 \%$ & $40 \%$ \\
\hline$[\mathrm{t}](0,407)$ & $169 / 749$ & $169 / 693$ & $615 / 1173$ & $554 / 1486$ & $554 / 1486$ & \\
& $23 \%$ & $24 \%$ & $52 \%$ & $37 \%$ & $37 \%$ & $72 \%$ \\
\hline
\end{tabular}

${ }^{a}$ La présente étude comprend aussi 74 sites de liaison avec [R] dont aucune n'est réalisée et 9 sites avec [p] dont une seulement (11\%), trop étroite, est réalisée par V. Le [p] défavorise fortement la liaison avec un poids de 0,262 . Encrevé (1988:77, note 11) nous renseigne que le [p] n'est pas représenté dans le corpus réduit des deux débats entre Giscard d'Estaing et Mitterrand.

${ }^{\mathrm{b}}$ Cette colonne pour la présente étude montre les chiffres après l'exclusion des liaisons catégoriques qui sont donc les chiffres sur lesquels Goldvarb X a basé le poids de chaque consonne.

${ }^{c}$ La colonne Ashby 1981A reproduit les chiffres qu'il a présentés lui-même tandis que la colonne Ashby 1981B présente les chiffres que j'ai recalculés moi-même pour exclure les liaisons considérées comme invariables, c'est-à-dire la liaison entre un pronom d'objet et un verbe et entre un pronom sujet et un verbe. On voit que ces liaisons invariables étaient très nombreuses pour la consonne $[\mathrm{n}]$.

${ }^{\mathrm{c}}$ Encrevé a trouvé que toutes les liaisons après [n] étaient soit invariables soit erratiques, alors il n'y a pas d'exemples de la consonne de liaison [n] dans son analyse de la liaison variable.

Pour atteindre notre but de déterminer l'importance relative de la consonne de liaison et de la fonction grammaticale des mots en liaison, il convient maintenant de déterminer la corrélation entre la fonction grammaticale et la consonne de liaison. Le Tableau 4 ci-dessous présente le taux de liaison de chaque consonne selon la fonction grammaticale et nous montre ainsi que le taux de liaison dépend presque entièrement de la fonction grammaticale et non pas de la consonne de liaison. La consonne de liaison [n], par exemple, n'apparaît que 55 fois dans notre corpus et après deux mots seulement, la préposition en et l'adverbe bien. Il semble donc que le taux de liaison du [n] est relativement élevé parce qu'il apparaît surtout dans la préposition en après laquelle la liaison est réalisée à une exception près. Les observations sur [z] sont encore plus révélatrices. Cette consonne apparaît soit dans des contextes presque invariables - après les adverbes très et plus, les prépositions chez, dans, sans, ou l'auxiliaire être-soit dans des contextes où elle n'est jamais prononcée. Même une fonction grammaticale qui était considérée comme variable en général, comme après la copule être, ne l'est presque pas pour la consonne [z]. On constate alors que le taux de liaison pour le $[\mathrm{z}]$ dans notre corpus dépend presque exclusivement de la proportion des sites de liaison presque invariable (19\%) contre les sites de liaison jamais réalisée (71\%). 
Tableau 4 : Le taux de liaison réalisée selon la fonction grammaticale et la consonne de liaison ${ }^{\mathrm{a}}$

\begin{tabular}{|c|c|c|c|}
\hline & {$[\mathrm{n}]$} & {$[\mathrm{t}]$} & {$[\mathrm{z}]$} \\
\hline $\begin{array}{l}\text { adv. très et plus } 49 / 51,96 \% \\
\text { très important }\end{array}$ & & & $\begin{array}{l}49 / 51,96 \% \\
9,6 \%\end{array}$ \\
\hline préposition $89 / 94,95 \%$ & $41 / 42,98 \%$ & & $48 / 52,92 \%$ \\
\hline dans une famille & $76,4 \%$ & & $9,8 \%$ \\
\hline total des liaisons fréquentes & $41 / 42,98 \%$ & & $97 / 103,94 \%$ \\
\hline $138 / 145,95 \%$ & $76,4 \%$ & & $19,4 \%$ \\
\hline être (aux.) 33/45, 73\% & & $21 / 27,78 \%$ & $12 / 18,67 \%$ \\
\hline il est arrivé & & $3,6 \%$ & $3,4 \%$ \\
\hline quand (conjonction) $36 / 52,69 \%$ & & $36 / 52,69 \%$ & \\
\hline quand elle téléphone & & $6,9 \%$ & \\
\hline c'est-à-dire 7/17, 41\% & & $7 / 17,41 \%$ & \\
\hline & & $2,3 \%$ & \\
\hline être (copule) 98/377, 26\% & & $95 / 344,28 \%$ & $3 / 33,9 \%$ \\
\hline c'est une langue & & $45,9 \%$ & $6,2 \%$ \\
\hline total des liaisons variables & & $159 / 440,28 \%$ & $15 / 51,29 \%$ \\
\hline $174 / 491,35 \%$ & & $58,7 \%$ & $9,6 \%$ \\
\hline nom pl. + adj. ou verbe $7 / 53,13 \%$ & & & $7 / 53,13 \%$ \\
\hline étudiants étrangers & & & $10,0 \%$ \\
\hline avoir (aux.) 3/25, 12\% & & $3 / 19,16 \%$ & $0 / 6,0 \%$ \\
\hline ils ont été & & $2,5 \%$ & $1,1 \%$ \\
\hline adverbe polysyllabique + bien, mieux & $5 / 13,38 \%$ & $0 / 55,0 \%$ & $2 / 27,7 \%$ \\
\hline moins & $23,6 \%$ & $7,3 \%$ & $5,1 \%$ \\
\hline verbe $8 / 298,3 \%$ & & $7 / 234,3 \%$ & $1 / 64,2 \%$ \\
\hline il y avait un animal & & $26,4 \%$ & $12,1 \%$ \\
\hline adv. négatif $0 / 76,0 \%$ & & & $0 / 76,0 \%$ \\
\hline pas informatisé & & & $14,3 \%$ \\
\hline mais, puis, alors $0 / 152,2 \%$ & & $0 / 1,0 \%$ & $0 / 151,0 \%$ \\
\hline mais après, puis ensuite & & & $28,4 \%$ \\
\hline total des liaisons rares & $5 / 13,38 \%$ & $10 / 309,7 \%$ & $10 / 377,3 \%$ \\
\hline $7 / 241,3 \%$ & $23,6 \%$ & $41,3 \%$ & $71,0 \%$ \\
\hline total des liaisons possibles & 55 & 749 & 531 \\
\hline $1335+9[\mathrm{p}](\mathrm{adv}),.+74[\mathrm{R}]($ verbe $)$ & & & \\
\hline
\end{tabular}


${ }^{a}$ Les chiffres après la fonction grammaticale sont le nombre de liaisons réalisées pour cette fonction divisé par le nombre de liaisons possibles suivi par le taux de liaison pour cette fonction. Dans les cases pour chaque consonne se trouvent ces mêmes chiffres sur la première rangée. Le pourcentage dans la deuxième rangée est le nombre total d'occurrences pour cette fonction divisé par le nombre total d'occurrences de liaisons possibles pour cette consonne. Par exemple, les 42 sites possibles de liaison pour [n] après une préposition représentent $76,4 \%$ du total de 55 sites possibles pour [n].

C'est seulement la consonne $[\mathrm{t}]$ qui affiche un comportement variable, surtout pour deux fonctions grammaticales, après quand et après être comme auxiliaire, copule, ou dans l'expression c'est-àdire. Ces catégories de liaison variable représentent $58,7 \%$ du total des sites possibles pour [t] tandis que les sites où la liaison n'est presque jamais réalisée représentent $41,3 \%$ du total. Alors on voit que le faible taux de liaison pour le [t] (23\%) est expliqué par son absence dans les liaisons presque toujours faites et sa présence dans les liaisons variables et presque jamais faites. Le [z] était la consonne latente dans beaucoup de sites où la liaison n'est presque jamais faite (71\%) mais elle était très présente aussi dans les liaisons presque toujours faites mais pas fréquente dans les liaisons variables. La plus grande différence entre le taux de liaison réalisée pour le $[\mathrm{z}]$ et le $[\mathrm{t}]$ est donc que les sites de liaison presque jamais faites représentent une plus grande proportion des sites possibles pour le $[\mathrm{z}]$ que pour le [t]. En plus nous constatons que le [t] est la seule consonne avec un comportement variable à quelques exceptions près. Cette constatation soulève la question intéressante de si la consonne [z] s'est associée dans l'esprit des locuteurs avec la liaison invariable_réalisée ou non-grâce à son apparence fréquente dans les liaisons invariablement faites et si la consonne [t] s'est alors associée avec la liaison variable, dans la mesure où quelques liaisons restent variables.

Les résultats présentés dans le Tableau 4, un tableau croisé de l'interaction entre la classe grammaticale et la consonne latente, soutiennent notre hypothèse que la fonction grammaticale est un facteur plus important que la consonne latente. On oserait même dire que c'est la classe grammaticale qui détermine les résultats pour la consonne latente. Cette idée est soutenue aussi par l'analyse binomiale de Goldvarb dit « step up step down » qui compare progressivement les groupes de facteurs pour déterminer leur importance relative. Dans l'analyse montante, dite « step up », dans laquelle Goldvarb analyse un groupe de facteurs et puis en ajoute un autre et un autre progressivement, la classe grammaticale a été retenue comme le facteur le plus important accompagné de deux autres facteurs, le locuteur individuel et l'âge. Dans l'analyse descendante, dite "step down », dans laquelle Goldvarb commence par tous les groupes de facteurs et puis les enlève progressivement, la classe grammaticale a été retenue encore une fois comme le facteur le plus important suivi par le locuteur individuel.

\subsection{La liaison variable et le sexe et l'âge des locuteurs}

En plus du facteur linguistique de la consonne de liaison, on peut analyser l'effet des facteurs diastratiques du sexe et de l'âge des locuteurs sur la liaison variable. Le Tableau 5 présente les résultats de la présente étude et aussi ceux des études de Malécot (1975) et d'Ashby (1981) pour le taux de liaison réalisée selon le sexe et l'âge des sujets parlants.

Nous voyons que les femmes ont un plus haut taux de liaison variable que les hommes dans l'étude de Malécot. Booij et De Jong (1987:1017) ont trouvé cette même tendance aussi dans leur analyse de la liaison dans certaines expressions, comme quand on est, souvent ensemble, et depuis un mois, prononcés dans une lecture et dans une liste de mots. Le taux de liaison réalisée était plus élevé chez les femmes pour 5 des 6 expressions analysées. Les hommes avaient un plus haut taux de liaison seulement dans souvent absent dans une liste de mots. L'étude d'Ashby a trouvé le contraire : que les hommes faisaient plus de liaisons que les femmes. Dans la présente étude on a l'impression que les femmes ont un taux de liaison légèrement plus élevé, mais une fois exclus les sites de liaison catégorique, le taux de liaison pour les deux sexes est presque identique, ce qui est confirmé par un poids aussi proche de 0,500 que possible et pour les hommes et pour les femmes. Il va presque sans 
dire que cette différence entre les sexes n'était pas statistiquement significative. Quant à l'âge, les trois études ont trouvé que les vieux avaient un plus haut taux de liaison que les jeunes. Booij et De Jong $(1987: 1017)$ ont trouvé cette même distinction chez des sujets parlants répartis en trois tranches d'âge après est, sont et suis dans des interviews et pour les expressions, depuis un mois, depuis un an, et pendant un moment, prononcées dans une lecture et dans une liste de mots. Le taux de liaison augmentait toujours avec l'âge sauf quand il était le même pour deux groupes et sauf dans le cas de depuis un an dans une liste de mots où l'âge au milieu a fait un bien plus grand pourcentage de liaisons (89\%) que les plus vieux (50\%). Dans la présente étude la différence entre le taux de liaison pour les jeunes et les vieux est statistiquement significative (à moins de 0,0001). Cette différence est d'autant plus significative puisque les jeunes ont fait plus d'années d'études que les vieux, un fait qui semblerait augmenter le taux de liaison chez les jeunes.

Tableau 5 : Le taux de liaison réalisée selon le sexe et l'âge des locuteurs

\begin{tabular}{|l|l|l|l|l|}
\hline & présente étude A & présente étude B & $\begin{array}{l}\text { Malécot } \\
1975: 169\end{array}$ & Ashby 1981:50 \\
\hline $\begin{array}{l}\text { les hommes } \\
(0,499)\end{array}$ & $\begin{array}{l}176 / 766 \\
23 \%\end{array}$ & $\begin{array}{l}176 / 542 \\
32,5 \%\end{array}$ & -- & $62 \%$ \\
\hline $\begin{array}{l}\text { les femmes } \\
(0,501)\end{array}$ & $162 / 652$ & $162 / 495$ & -- & $519 / 1447$ \\
\hline les jeunes & $25 \%$ & $32,7 \%$ & $67 \%$ & $36 \%$ \\
$(0,403)$ & $111 / 634$ & $111 / 459$ & -- & $553 / 1735$ \\
\hline $\begin{array}{l}\text { les vieux } \\
(0,577)\end{array}$ & $18 \%$ & $24 \%$ & $63 \%$ & $32 \%$ \\
\hline
\end{tabular}

${ }^{\mathrm{a}}$ Comme dans le Tableau 3, la présente étude A comprend tous les sites possibles de liaisons et la présente étude $\mathrm{B}$ présente les résultats après l'exclusion des liaisons catégoriquement non réalisées.

${ }^{\mathrm{b}}$ Les jeunes dans la présente étude ont entre 22 et 32 ans et les vieux ont entre 40 et 65 ans. Dans l'étude d'Ashby les jeunes sont encore plus jeunes ayant entre 14 et 21 ans et les vieux ont entre 51 et 64 ans. Le corpus de Malécot comprend des locuteurs de 20 ans jusqu'à 69 ans repartis en quatre tranches d'âge, mais pour ce tableau j'ai choisi seulement les deux tranches d'âge qui étaient les plus comparables à ceux des autres études-les jeunes entre 20 et 29 ans et les vieux entre 50 et 59 ans.

En accord avec notre objectif de déterminer l'importance relative des facteurs diastratiques, le sexe et l'âge, et la fonction grammaticale, le Tableau 6 est un tableau croisé qui présente les proportions des liaisons faites et des liaisons possibles de la fonction grammaticale selon le sexe et l'âge des sujets parlants. Nous cherchons à déterminer si la différence entre les deux sexes est les deux âges est due au hasard à la proportion de différentes fonctions grammaticales pour la liaison dans le corpus ou si elle est basée sur de vraies différences de comportement linguistique entre les deux groupes sociaux.

Les résultats montrent que les proportions des liaisons fréquentes, variables et rares sont extrêmement proches pour les hommes et les femmes, ce qui est vrai aussi pour leur taux de liaison pour les différentes classes grammaticales. Les seules différences notables entre les deux sexes sont le plus haut taux de liaison chez les hommes pour c'est-à-dire (50\% contre $29 \%)$ et après un nom pluriel $(19 \%$ contre $8 \%)$, mais ce sont des catégories où il y a en tous cas peu d'exemples. Les femmes ont un plus haut taux de liaison après un verbe (3\% contre $1 \%)$, mais cette différence se repose en fin de compte sur 6 liaisons réalisées par les femmes contre 2 par les hommes. Il faut conclure, dans ce corpus du moins, que le sexe du locuteur n'a aucun effet sur la réalisation de la liaison. 
Tableau 6: Le taux de liaison réalisée selon la fonction grammaticale et le sexe et l'âge

\begin{tabular}{|c|c|c|c|c|}
\hline & les hommes & les femmes & les jeunes & les vieux \\
\hline $\begin{array}{l}\text { adv. très et plus } 49 / 51,96 \% \\
\text { très important }\end{array}$ & $\begin{array}{l}20 / 21,95 \% \\
2,7 \%\end{array}$ & $\begin{array}{l}29 / 30,97 \% \\
4,6 \%\end{array}$ & $\begin{array}{l}13 / 13,100 \% \\
2,1 \%\end{array}$ & $\begin{array}{l}36 / 38,95 \% \\
4,8 \%\end{array}$ \\
\hline $\begin{array}{l}\text { préposition } 89 / 94,95 \% \\
\text { dans une famille }\end{array}$ & $\begin{array}{l}46 / 49,94 \% \\
6,4 \% \\
\end{array}$ & $\begin{array}{l}43 / 45,96 \% \\
6,9 \% \\
\end{array}$ & $\begin{array}{l}51 / 53,96 \% \\
8,4 \% \\
\end{array}$ & $\begin{array}{l}38 / 41,93 \% \\
5,2 \% \\
\end{array}$ \\
\hline $\begin{array}{l}\text { total des liaisons fréquentes } \\
138 / 145,95 \%\end{array}$ & $\begin{array}{l}66 / 70,94 \% \\
9,1 \% \\
\end{array}$ & $\begin{array}{l}72 / 75,96 \% \\
11,5 \% \\
\end{array}$ & $\begin{array}{l}64 / 66,97 \% \\
10,4 \% \\
\end{array}$ & $\begin{array}{l}74 / 79,94 \% \\
10,1 \% \\
\end{array}$ \\
\hline $\begin{array}{l}\text { etre (aux.) 33/45, 73\% } \\
\text { il est arrivé }\end{array}$ & $\begin{array}{l}20 / 27,74 \% \\
3,5 \%\end{array}$ & $\begin{array}{l}13 / 18,72 \% \\
2,8 \%\end{array}$ & $\begin{array}{l}7 / 15,47 \% \\
2,7 \%\end{array}$ & $\begin{array}{l}26 / 30,87 \% \\
3,8 \%\end{array}$ \\
\hline $\begin{array}{l}\text { quand (conj.) 36/52, 69\% } \\
\text { quand elle téléphone }\end{array}$ & $\begin{array}{l}22 / 34,65 \% \\
4,4 \%\end{array}$ & $\begin{array}{l}14 / 18,78 \% \\
2,8 \%\end{array}$ & $\begin{array}{l}11 / 22,50 \% \\
3,5 \%\end{array}$ & $\begin{array}{l}25 / 30,83 \% \\
3,8 \% \\
\end{array}$ \\
\hline c'est-à-dire 7/17, 41\% & $\begin{array}{l}5 / 10,50 \% \\
1,3 \%\end{array}$ & $\begin{array}{l}2 / 7,29 \% \\
1,1 \%\end{array}$ & $\begin{array}{l}1 / 5,20 \% \\
0,8 \%\end{array}$ & $\begin{array}{l}6 / 12,50 \% \\
1,5 \%\end{array}$ \\
\hline $\begin{array}{l}\text { être (copule) 98/377, 26\% } \\
\text { c'est une langue }\end{array}$ & $\begin{array}{l}51 / 195,26 \% \\
25,5 \%\end{array}$ & $\begin{array}{l}47 / 182,26 \% \\
27,9 \%\end{array}$ & $\begin{array}{l}24 / 190,13 \% \\
30,0 \%\end{array}$ & $\begin{array}{l}74 / 187,40 \% \\
23,9 \% \\
\end{array}$ \\
\hline $\begin{array}{l}\text { total des liaisons variables } \\
174 / 491,35 \%\end{array}$ & $\begin{array}{l}98 / 266,37 \% \\
34,7 \%\end{array}$ & $\begin{array}{l}76 / 225,34 \% \\
34,5 \%\end{array}$ & $\begin{array}{l}43 / 232,19 \% \\
36,6 \%\end{array}$ & $\begin{array}{l}131 / 259,51 \% \\
33,0 \%\end{array}$ \\
\hline $\begin{array}{l}\text { nom pl. + adj. / verbe } \\
7 / 53,13 \% \text { étudiants étrangers }\end{array}$ & $\begin{array}{l}5 / 27,19 \% \\
3,5 \% \\
\end{array}$ & $\begin{array}{l}2 / 26,8 \% \\
4,0 \%\end{array}$ & $\begin{array}{l}1 / 18,6 \% \\
2,8 \%\end{array}$ & $\begin{array}{l}6 / 35,17 \% \\
4,5 \%\end{array}$ \\
\hline $\begin{array}{l}\text { avoir (aux.) } 3 / 25,12 \% \\
\text { ils ont été }\end{array}$ & $\begin{array}{l}1 / 10,10 \% \\
1,3 \%\end{array}$ & $\begin{array}{l}2 / 15,13 \% \\
2,3 \%\end{array}$ & $\begin{array}{l}0 / 5,0 \% \\
0,8 \%\end{array}$ & $\begin{array}{l}3 / 20,15 \% \\
2,6 \%\end{array}$ \\
\hline $\begin{array}{l}\text { adverbe polysyllabique }+ \text { bien, } \\
\text { mieux, moins } 8 / 104,8 \%\end{array}$ & $\begin{array}{l}4 / 55,7 \% \\
7,2 \%\end{array}$ & $\begin{array}{l}4 / 49,8 \% \\
7,5 \%\end{array}$ & $\begin{array}{l}2 / 57,4 \% \\
9,0 \%\end{array}$ & $\begin{array}{l}6 / 47,13 \% \\
6,0 \% \\
\end{array}$ \\
\hline $\begin{array}{l}\text { verbe } 8 / 372,2 \% \\
\text { il y avait un animal }\end{array}$ & $\begin{array}{l}2 / 194,1 \% \\
25,3 \% \\
\end{array}$ & $\begin{array}{l}6 / 178,3 \% \\
27,3 \% \\
\end{array}$ & $\begin{array}{l}1 / 165,1 \% \\
26,0 \%\end{array}$ & $\begin{array}{l}7 / 207,3 \% \\
26,4 \% \\
\end{array}$ \\
\hline $\begin{array}{l}\text { adv. négatif } 0 / 76,0 \% \\
\text { pas informatisé }\end{array}$ & $\begin{array}{l}0 / 42,0 \% \\
5,5 \%\end{array}$ & $\begin{array}{l}0 / 34,0 \% \\
5,2 \%\end{array}$ & $\begin{array}{l}0 / 36,0 \% \\
5,7 \%\end{array}$ & $\begin{array}{l}0 / 40,0 \% \\
5,1 \%\end{array}$ \\
\hline $\begin{array}{l}\text { mais, puis, alors } 0 / 152,2 \% \\
\text { mais après, puis ensuite }\end{array}$ & $\begin{array}{l}0 / 102,1 \% \\
13,3 \% \% \\
\end{array}$ & $\begin{array}{l}0 / 50,7 \% \\
7,7 \% \\
\end{array}$ & $\begin{array}{l}0 / 55,0 \% \\
8,7 \% \\
\end{array}$ & $\begin{array}{l}0 / 97,4 \% \\
12,4 \% \\
\end{array}$ \\
\hline $\begin{array}{l}\text { total des liaisons rares } \\
26 / 782,3 \% \\
\end{array}$ & $\begin{array}{l}12 / 430,3 \% \\
56,1 \% \\
\end{array}$ & $\begin{array}{l}14 / 352,4 \% \\
54,0 \% \\
\end{array}$ & $\begin{array}{l}4 / 336,1 \% \\
53,0 \% \\
\end{array}$ & $\begin{array}{l}22 / 446,5 \% \\
56,9 \\
\end{array}$ \\
\hline $\begin{array}{l}\text { total des liaisons possibles } \\
1418\end{array}$ & 766 & 652 & 634 & 784 \\
\hline
\end{tabular}


*Comme pour le Tableau 4, les premiers chiffres dans chaque case sont le nombre de liaisons faites pour cette fonction grammaticale pour chaque groupe divisé par le nombre de liaisons possibles et suivis alors par le pourcentage. La deuxième ligne de chaque case indique le pourcentage des liaisons possibles pour cette fonction grammaticale sur le nombre de liaisons possibles totales pour ce groupe social.

L'analyse binomiale «step up step down » de Goldvarb X soutient les conclusions déjà tirées des pourcentages des liaisons réalisées par les hommes et les femmes et les jeunes et les vieux présentés dans le Tableau 6. Comme on a vu ci-dessus (en 3.2) les facteurs qui ont été retenus comme importants après la classe grammaticale était le locuteur individuel et l'âge des locuteurs. Puisque l'analyse de Goldvarb X a indiqué que l'individu était même plus important que son appartenance à une certaine tranche d'âge, le Tableau 7 présente le taux de liaison et le poids relatif pour chaque locuteur. Les taux de liaison vont de $13 \%$ à $40 \%$ pour les jeunes et de $26 \%$ à $55 \%$ pour les vieux. On voit que les jeunes qui ont les taux de liaison les plus élevés sont plus âgés que les autres; C âgée de 32 ans a un taux de liaison de $40 \%$ et $\mathrm{F}$ âgée de 28 ans a un taux de $32 \%$. On se demande alors s'il y a des différences d'âge plus subtiles qu'un échantillon plus grand de locuteurs permettrait de découvrir. En tous cas quatre locuteurs âgés (T, U, W, Y) ont des taux de liaison plus bas que celui de la locutrice $\mathrm{C}$, mais les quatre taux de liaison les plus bas se trouvent chez les jeunes $(\mathrm{D}, \mathrm{E}, \mathrm{A}, \mathrm{H})$ et les trois taux les plus élevés $(\mathrm{X}, \mathrm{S}, \mathrm{Z})$ se trouvent chez les vieux. Il y a certainement une corrélation entre l'âge et le taux de liaison mais il faut tenir compte aussi des différences individuelles importantes.

Tableau 7 : Le taux de liaison réalisée selon le locuteur individuel

\begin{tabular}{|c|c|c|c|c|c|}
\hline & locuteur & âge & $\begin{array}{l}\text { liaison } \\
\text { faites/liaisons } \\
\text { possibles }\end{array}$ & $\%$ & poids \\
\hline \multirow{4}{*}{$\begin{array}{l}\text { jeunes } \\
\text { femmes }\end{array}$} & $\mathrm{A}$ & 25 & $13 / 68$ & $19 \%$ & 0,340 \\
\hline & $\mathrm{B}$ & 25 & $13 / 48$ & $27 \%$ & 0,448 \\
\hline & $\mathrm{C}$ & 32 & $18 / 45$ & $40 \%$ & 0,593 \\
\hline & $\mathrm{D}$ & 22 & $6 / 45$ & $13 \%$ & 0,252 \\
\hline \multirow{4}{*}{$\begin{array}{l}\text { jeunes } \\
\text { hommes }\end{array}$} & $\mathrm{E}$ & 25 & $10 / 61$ & $16 \%$ & 0,300 \\
\hline & $\mathrm{F}$ & 28 & $19 / 59$ & $32 \%$ & 0,509 \\
\hline & $\mathrm{G}$ & 24 & $20 / 68$ & $29 \%$ & 0,476 \\
\hline & $\mathrm{H}$ & 26 & $12 / 65$ & $19 \%$ & 0,331 \\
\hline \multirow{4}{*}{$\begin{array}{l}\text { femmes } \\
\text { âgées }\end{array}$} & $S$ & 55 & $36 / 68$ & $53 \%$ & 0,710 \\
\hline & $\mathrm{T}$ & 56 & $32 / 95$ & $34 \%$ & 0,526 \\
\hline & $\mathrm{U}$ & 53 & $15 / 53$ & $28 \%$ & 0,463 \\
\hline & $\mathrm{V}$ & 40 & $29 / 73$ & $40 \%$ & 0,590 \\
\hline \multirow[t]{4}{*}{ hommes âgés } & $\mathrm{W}$ & 56 & $23 / 87$ & $26 \%$ & 0,440 \\
\hline & $\mathrm{X}$ & 65 & $33 / 84$ & $39 \%$ & 0,585 \\
\hline & $\mathrm{Y}$ & 55 & $40 / 73$ & $55 \%$ & 0,725 \\
\hline & $\mathrm{Z}$ & 55 & $19 / 45$ & $42 \%$ & 0,614 \\
\hline
\end{tabular}

\section{Conclusion}

L'analyse présentée ici de la liaison variable dans un corpus récent du français parlé méridional a permis de nouveau d'apprécier la complexité de ce phénomène phonologique qui a fait l'objet de nombreuses études. (Voir surtout la bibliographie dans Durand et Lyche 2008). Notre objectif spécifique dans cette étude était de déterminer l'importance relative de la fonction grammaticale par rapport à un autre facteur linguistique, la consonne de liaison, et deux facteurs diastratiques, le sexe et l'âge. À l'aide d'un tableau croisé de la classe grammaticale du mot liaisonnant et un autre facteur et une analyse statistique et binomiale, nous avons pu confirmer que la consonne de liaison 
tout aussi bien que la classe grammaticale a un effet statistiquement significatif sur le taux de liaison. Néanmoins, il paraît que la classe grammaticale est un facteur plus important que la consonne de liaison. Le Tableau 4 a montré que le taux de liaison pour chaque consonne latente dépend presque entièrement de la fonction grammaticale des mots en liaison et l'analyse binomiale a retenu la classe grammaticale comme facteur important et non pas la consonne de liaison. Il serait intéressant de déterminer dans l'avenir si ce serait le cas aussi pour d'autres facteurs linguistiques, tels que la longueur du mot (Booij et De Jong 1987 :1013, Pagliano et Laks 2005 :4), la fréquence du mot, et la nature des phonèmes avant et après la consonne de liaison (Booij et De Jong 1987 :1013). La corrélation entre la fonction grammaticale et les facteurs diastratiques s'est avérée plus complexe. On a pu voir facilement que le sexe du locuteur n'a aucun effet sur le taux de liaison, mais que l'âge semble être un facteur important. Il était particulièrement intéressant de comparer le comportement différent des deux tranches d'âge pour la liaison selon la classe grammaticale du mot liaisonnant. Il semble que la tendance actuelle est de réduire la variabilité de la liaison. Les jeunes ont tendance à faire presque toujours les liaisons les plus fréquentes et de faire de moins en moins les liaisons autrefois variables et les liaisons les plus rarement faites. Nous voyons donc que notre hypothèse est soutenue à moitié par ces résultats. Il est vrai que la classe grammaticale est le facteur le plus important pour la réalisation de la liaison, mais il n'est pas vrai que les différentes proportions de différentes classes grammaticales de liaison affectent les résultats pour les autres facteurs, surtout si on s'occupe d'exclure les liaisons catégoriques de son analyse. Toutefois, vu l'importance de la classe grammaticale, il semble préférable de comparer les résultats pour la même classe grammaticale au moment de tester les facteurs diastratiques, comme on a fait dans le Tableau 6. Pour les futures recherches, il serait utile d'analyser en plus grand détail les liaisons qui sont toujours restées variables, c'est-à-dire la liaison après être et après quand. Il nous paraît surtout prometteur de faire une analyse pragmatique de ces liaisons pour voir, comme a suggéré Encrevé (1988:258), si le style de parole à différents moments de la conversation, ou le micro-style, explique leur réalisation ou non réalisation. Il semble possible que la liaison, étant de nos jours la forme marquée, puisse servir à mettre en relief l'idée présentée par le mot liaisonnant. Pour ne citer qu'un seul exemple on trouve que le locuteur Y prononce le [t] de c'est dans c'est un dialecte quand il souligne le contraste entre un dialecte et une langue mais qu'il ne le prononce pas dans l'expression c'est en kilomètres dans laquelle il ne fait aucun contraste. On voudrait voir donc dans l'avenir si jamais la liaison sert à renforcer l'idée exprimée par le mot liaisonnant.

\section{Références bibliographiques}

Ågren, J. (1973). Enquête sur quelques liaisons facultatives dans le français de conversation radiophonique. Uppsala : Acta Universitatis Upsaliensis.

Ashby, W. J. (1981). French liaison as a sociolinguistic phenomenon. Linguistic symposium on Romance Languages, ed. par W. W. Cressey et D. J. Napoli, 46-57. Washington, DC : Georgetown University Press.

Armstrong, N. (2001). Social and Stylistic Variation in Spoken French: A comparative approach. Amsterdam/Philadelphia : John Benjamins.

Booij, G. et De Jong, D. (1987). The domain of liaison : Theories and data. Linguistics 25 :1005-25.

De Jong, D., Poll, E., et Woudman, W. (1981). La liaison : l'influence sociale et stylistique sur l'emploi de la liaison dans le français parlé à Tours. Thèse de master inédite, Université de Groningen.

Delattre, P. (1947). La liaison en français, tendances et classification. The French Review 8 :148-57.

Delattre, P. (1966). Studies in French and Comparative Phonetics. La Haye : Mouton.

Durand, J. et Lyche, C. 2008. French liaison in the light of corpus data. French Language Studies 18:33-66.

Encrevé, P. (1988). La liaison avec et sans enchaînement. Paris : Éditions du Seuil. 
Green, J. N. et Hintze, M-A. (1990). Variation and change in French linking phenomena. Variation and change in French: Essays presented to Rebecca Posner on the occasion of her sixtieth birthday, ed. par J. N. Green et W. Ayres-Bennett, 61-88. London/New York : Routledge.

Lodge, A., Armstrong, N., Ellis, Y. M. L., et Shelton, J. F. (1997). Exploring the French Language. London : Arnold.

Malécot, A. (1975). French Liaison as a Function of Grammatical, Phonetic and Paralinguistic Variables. Phonetica, 32, 161-79.

Pagliano, C. et Laks, B. (2005). Problématique de la liaison dans l'analyse d'un corpus de français oral actuel. Français fondamental, corpus oraux, contenus d'enseignement. 50 ans de travaux et d'enjeux, 8-10 décembre 2005, École Normale Supérieure Lettres et Science Humaines, Lyon, France. http://colloqueff.ens-lsh.fr/pdf/Laks_Bernard_Pagliano_Claudine.pdf.

Sankoff, D., Tagliamonte, S., et Smith, E. (2005). Goldvarb X: A multivariate analysis application. http://individual.utoronto.ca/tagliamonte/Goldvarb/GV_index.htm.

Smith. A. (1996). A diachronic study of French variable liaison. Thèse MLitt. inédite, Université de Newcastle upon Tyne.

Smith. A. (1998). French liaison : a proposed simplification. Francophonie $17: 11-14$.

Tagliamonte, S. A. (2006). Analysing sociolinguistic variation. Cambridge : Cambridge University Press.

Valdman, A. (1993). Bien Entendu! Introduction à la prononciation française. Englewood Cliffs, NJ : Prentice Hall.

\footnotetext{
${ }^{1}$ Je tiens à remercier mon collègue Chad Howe pour son aide avec Goldvarb X.

${ }^{2}$ Cet échantillon de 16 locuteurs a été tiré d'un corpus plus grand qui comprend 33 conversations avec 40 locuteurs, dont 5 jeunes femmes du sud et 4 jeunes femmes du nord, 7 jeunes hommes du sud et 1 du nord, 9 femmes âgées du sud et 6 du nord, et 7 hommes âgés du sud et 1 du nord. Parmi ces locuteurs 22 ont été enregistrés à Montpellier en juillet 2005 et 18 à Rognes en mai 2006. Il m'est un grand plaisir de remercier les personnes qui m'ont aidée à constituer ce corpus. Debbie Bell, directrice du programme UGA en France, et mes cinq étudiants, Jeanne Brackin, Shanye Crawford, Chase Curry, Leslie Rogers, et Emily Wallace, m'ont aidée à faire les enregistrements à Montpellier. L'été suivant Janet Nicotri m'a aidée à trouver les sujets parlants enregistrés à Rognes et m'a hébergée pendant que je faisais ces enregistrements et le programme « Senior Teaching Fellows » à l'Université de Géorgie a financé ce voyage de recherche. Finalement je tiens à remercier du fond du cœur tous mes sujets parlants pour leur générosité et hospitalité.
}

${ }^{3}$ Donc nous n'avons pas considéré les pauses comme des sites possibles de liaison puisque la liaison sans enchaînement, présentée par Encrevé (1988) et discutée plus tard par Durand et Lyche $(2008: 48-52)$, n'est représentée que par un seul exemple dans notre corpus : entre quand et $i l$ dans la phrase Et ça a pris une grande place quand il y a un tout prononcé par le locuteur W. Deux fois la consonne latente [t] de c'est est réalisée devant euh par le locuteur X.

${ }^{4}$ Nous avons dû exclure et de la comparaison avec les études antérieures et de l'analyse variationniste quatre liaisons possibles. Deux étaient le seul exemple d'un certain mot liaisonnant, la préposition vers (par V) et le pronom négatif rien dans l'expression rien à voir (par E), dont aucune n'a été faite, et une liaison après en qui était difficile à entendre, en autre langue (par S). On a exclu également le seul exemple dans ce corpus d'une fausse liaison, tellement agréable prononcé avez [z] comme consonne de liaison, donc comme [tzl $\therefore$ mãzagReabl], par T. C'est d'ailleurs le seul exemple d'une liaison réalisée après un adverbe polysyllabique dans tout le corpus. On a choisi de compter comme site de liaison les deux exemples du verbe mettent suivi par une voyelle, ils mettent un gros chiffon et ils mettent une pièce. Bien que la consonne finale du verbe sans liaison et la consonne de liaison soient toutes les deux [t], la réalisation du $e$ muet du verbe par un locuteur du sud permettrait d'entendre la consonne de liaison. Pourtant aucune de ces liaisons n'a été réalisée.

${ }^{5}$ Il convient de noter qu'il était nécessaire d'exclure les liaisons possibles avec la consonne [R] de l'analyse Goldvarb X aussi puisque le [R] n'a jamais été prononcé en liaison.

${ }^{6}$ Encrevé (1988:65) a rapporté les pourcentages de liaison réalisée après quelques mots invariables monosyllabiques qui ne rentrent pas dans le Tableau 2 mais qui ne sont pas sans intérêt. Dans deux débats politiques entre 1974 et 
1981, Valéry Giscard d'Estaing et François Mitterrand ont toujours fait la liaison après dans, sans, très, quand et dont. Seul Mitterrand a toujours fait la liaison après plus tandis que le taux de liaison après ce mot pour Giscard d'Estaing était de $67 \%$ en 1974 et de $50 \%$ en 1981. La liaison était variable seulement après mais et pas. Le taux de liaison après mais et pas était très bas chez Giscard d'Estaing (de $13 \%$ et $8 \%$ après mais et de $17 \%$ et $37 \%$ après pas) et plus haut chez Mitterrand (de $100 \%$ et $40 \%$ après mais et de $63 \%$ et $91 \%$ après pas).

${ }^{7}$ Les seules liaisons qui n'ont pas été faites après très ou plus sont un exemple sur 36 de très (très important par Y) et un exemple sur 14 de plus (plus éloigné par U). Après une préposition les liaisons non réalisées sont 3 exemples sur 40 après dans (dans un centre par $\mathrm{V}$, dans une famille par $\mathrm{U}$, et dans une administration par $\mathrm{W}$ ), un exemple sur 42 après en (en été par E), et un exemple de chez (chez un ami par $\mathrm{H}$ ) contre 5 liaisons réalisées (toutes dans l'expression chez eux). Il est intéressant que 5 de ces 7 non réalisations aient été faites par des locuteurs âgés bien que, comme nous le verrons plus tard, le groupe de locuteurs âgés produise un plus haut taux de liaison en général. 